\title{
Sparse Correlated Diffusion Imaging: A New Computational Diffusion MRI Modality for Prostate
}

Cancer Detection

Farzad Khalvati, Junjie Zhang, Sameer Baig, Masoom A. Haider

Dept. of Medical Imaging, Sunnybrook Research Institute

University of Toronto, Toronto, ON, Canada

Alexander Wong

Dept. of Systems Design Engineering, University of Waterloo

Waterloo, ON, Canada

\section{Abstract}

Diffusion weighted imaging (DWI) is a promising magnetic resonance imaging (MRI) modality with wide applications in diagnosis of different types of diseases such as prostate cancer. DWI provides a large amount of imaging data which often makes it difficult to interpret accurately, mainly due to the fact that much of information in diffusion imaging cannot be deciphered by human experts alone. Computational diffusion MRI (CD-MRI) aims to leverage computational means to generate imagery from diffusion signals which are easier to interpret by human experts. Recently, a new CD-MRI modality called correlated diffusion imaging (CDI) has been proposed which takes advantage of the joint correlation of diffusion signal attenuation across multiple gradient pulse strengths and timings to improve the separability of cancerous and healthy tissues. In this paper, we propose a new CD-MRI modality called Sparse CDI (sCDI) where an optimally sparse subset of diffusion signals contributes to the formation of the final diffusion signal leading to further separation of cancerous and healthy tissue in prostate gland compared to CDI and conventional DWI.

\section{Introduction}

Canadian Cancer Society indicates that 1 in 8 Canadian men will experience prostate cancer in their lifetimes [1]. Although it is one of the most common types of cancer, prostate cancer has shown to have high 5-year survival rate if diagnosed early [1]. As a result, early and accurate detection of prostate cancer is key in achieving high quality of lives for patients.

As part of multi-parametric MRI (mpMRI), diffusion-weighted imaging (DWI) has shown promise in accurate diagnosis of prostate cancer. Apparent diffusion coefficient (ADC) derived from DWI has been found to be able to predict pathology results (i.e., Gleason score) and clinically significant prostate cancer as well as differentiate between low-risk, intermediate-risk, and high-risk prostate cancer [2] (Figure 2-a). Even with the standardization of mpMRI reporting (PI-RADS [3]), the difficulty in interpreting mpMRI and particulary DWI leads to inter-observer variability among clinicians [4], which reduces the overall accuracy of diagnosis.

Computational diffusion MRI (CD-MRI) modalities aim to leverage computational means to generate imagery from diffusion signals which are easier to interpret by human experts. This is accomplished by generating imagery that improves the delineation between cancerous tissue from healthy tissue in a given organ (e.g., prostate). Recently, a new CD-MRI modality called correlated diffusion imaging (CDI) was introduced that takes advantage of joint correlation in diffusion signal attenuation across multiple gradient pulse strengths and timings to improve the delineation of cancerous and healthy tissue in prostate [5]. CDI combines different diffusion signals through a signal mixing function where local correlation of signal attenuations across different gradient pulse strengths and timings is computed [5] (Figure 2-b). It has been shown that this leads to improved delineation of cancerous and healthy tissue in prostate gland $[5,6,7,8]$. A different configuration of CDI (dualstage CDI or D-CDI) was also introduced to include more anatomical information of the prostate gland making it easier for visual assessment [9]. This was done by incorporating an additional signal mixing stage between the correlated diffusion signal from the first signal mixing stage and an auxiliary diffusion signal or T2-weighed image [9].

In both CDI and D-CDI, all diffusion signals contribute to the final CDI signal equally. In an attempt to optimize the contribution of each diffusion signal, a weighted CDI was introduced where each signal's contribution to the final CDI was controlled and optimized by a coefficient $[10,11]$. It was shown that the optimized CDI further improved the separability of cancerous and healthy tissue in prostate gland [10, 11].

In this paper, we introduce and explore a new CD-MRI modality called Sparse CDI (sCDI), where an optimally sparse subset of diffusion signals is leveraged to further improve the separability of cancerous and healthy regions in prostate. The goal is to find an optimally sparse subset of diffusion signals which yields the best results in terms of separability of cancerous and healthy tissue in the prostate gland.

\section{METHODOLOGY}

Before describing the methodology behind sparse correlated diffusion imaging ( $(\mathrm{CDI})$, let us first briefly describe the concept of correlated diffusion imaging (CDI) to provide the fundamentals behind sCDI. In the CDI imaging process, axial single-shot echo-planar sequences with two gradient pulses of equal magnitude (one pulse in each side of the $180^{\circ}$ pulse to dephase and rephase the spins, respectively) are used to obtain multiple signal acquisitions using a set of different configurations of gradient pulse strengths and timings, resulting in a set of $n$ acquired diffusion signals $S_{1}, S_{2}, \ldots, S_{n}$. These acquired signals are then mixed together to form a final signal using the following signal mixing function $C(\underline{x})$ for characterizing local signal attenuation correlation, which is parameterized by diffusion range defined by $\left[q_{\alpha}, q_{\beta}\right]$ and is defined as [5]:

$$
\begin{array}{r}
C_{\left[q_{\alpha}, q_{\beta}\right]}(\underline{x})=\int \ldots \int S_{q_{\alpha}}(\underline{x}) \ldots S_{q_{\beta}}(\underline{x}) \\
f\left(S_{q_{\alpha}}(\underline{x}), \ldots, S_{q_{\beta}}(\underline{x}) \mid V(\underline{x})\right) d S_{q_{\alpha}}(\underline{x}) \ldots d S_{q_{\beta}}(\underline{x})
\end{array}
$$

where $\underline{x}$ denotes spatial location, $S$ denotes the acquired signal, $f$ denotes the conditional joint probability density function, and $V(\underline{x})$ denotes the local sub-volume around $\underline{x}$. It can be observed in Equation 1 that all diffusion signals within the diffusion range $\left[q_{\alpha}, q_{\beta}\right]$ contribute equally to produce the final signal. In the proposed sparse correlated diffusion imaging (sCDI), we explore the notion that an optimally sparse subset of the diffusion signals within 
the diffusion range could potentially lead to improved delineation between prostate cancer tissue and healthy tissue.

In SCDI, let $\underline{R}=\left[R_{\alpha} \ldots R_{\beta}\right]$ be a sparse sampling vector, where $R_{i} \in\{0,1\}$ denotes a binary indicator of whether a particular diffusion signal is sampled. Incorporating $\underline{R}$, the signal mixing function $C(\underline{x})$ can be reformulated as:

$$
\begin{gathered}
C_{\left[q_{\alpha}, q_{\beta}\right], \underline{R}}(\underline{x})=\int \ldots \int S_{q_{\alpha}}(\underline{x})^{R_{\alpha}} \ldots S_{q_{\beta}}(\underline{x})^{R_{\beta}} \\
f\left(S_{q_{\alpha}}(\underline{x}), \ldots, S_{q_{\beta}}(\underline{x}) \mid V(\underline{x})\right) d S_{q_{\alpha}}(\underline{x}) \ldots d S_{q_{\beta}}(\underline{x})
\end{gathered}
$$

Since we wish to find the optimal sparse subset of diffusion signals within the diffusion range that yields the greatest delineation between prostate cancer tissue and healthy tissue, we incorporate Equation 2 into the following optimization problem, where the goal is to obtain the sparse sampling vector $\underline{R}$ and the associated $\mathrm{sCDI}$ signal $C$ that maximizes the area under the Receiver Operator Characteristic curve (denoted by $A_{z}$ ), with the condition that the number of non-zero elements in $\underline{R}$ must be equal to a sparsification factor $m$ :

$$
\{C, \underline{R}\}=\arg \max _{\{C, \underline{R}\}} A_{z}(C, \underline{R}) \quad \text { s.t. } \quad \sum_{i \in \underline{R}} R_{i}=m
$$

For this study, $\left[q_{\alpha}, q_{\beta}\right]$ was set at $\left[1300 \mathrm{~s} / \mathrm{mm}^{2}, 2200 \mathrm{~s} / \mathrm{mm}^{2}\right]$, and $V$ was defined as a $7 \mathrm{~mm}^{3}$ spatial sub-volume for assessment purposes as it was found to provide good tissue delineation, and the sparsification factor set to $m=3$. These diffusion-weighed images of b-values of $\left[1300 \mathrm{~s} / \mathrm{mm}^{2}, 2200 \mathrm{~s} / \mathrm{mm}^{2}\right]$ were computationally created using a Bayesian model with the least squares estimation technique using acquired diffusion images of $[0,400,600$, and $1000 \mathrm{~s} / \mathrm{mm}^{2}$ ] [12].

To maximize $A_{z}$ and find the optimal sparse sampling vector $\underline{R}$, a training data was used in which the tumourous regions had been marked by a radiologist as ground truth.

\section{EXPERIMENTAL SETUP}

In order to evaluate the performance of the proposed SCDI, diffusion imaging was performed on 17 patients. The images were acquired using a Philips Achieva 3.0T machine at Sunnybrook Health Sciences Centre, Toronto, Ontario, Canada. All data was obtained under the local institutional research ethics board.

To find the optimally sparse subset of diffusion signals (i.e., the optimal sparse sampling vector $\underline{R}$ ), diffusion signals from 9 patients with PI-RADS scores 4 and 5 where the tumour sites were annotated and contoured by an experienced radiologist were analyzed. PI-RADS scores of 4 and 5 indicate the high likelihood of clinically significant cancer [3].

SCDI was performed based on the optimal sparse sampling vector $\underline{R}$ using Equation 3. Eight patient cases with pathologyconfirmed significant prostate cancer (Gleason score 6 and above) were used to evaluate SCDI performance. The performances of sCDI, original CDI, and ADC (derived from DWI) were calculated using the area under ROC curve $\left(A_{z}\right)$ compared to histology results for these eight patient cases.

\section{Results}

Table 1 shows the area under ROC curve for for ADC, CDI, and sCDI. As it can be seen, the area under ROC curve for SCDI is
0.92, which is higher than those for ADC (0.86) and CDI (0.88). The results for $S C D I$ is also significantly different than those for $A D C$ and $C D I$ with $P<0.05$ for sCDI compared to CDI and ADC, indicating statistical significance.

Table 1: Evaluation of SCDI results for prostate cancer detection

\begin{tabular}{|c|c|c|c|}
\hline Modality & ADC & CDI & SCDI \\
\hline \hline Area under ROC curve & 0.86 & 0.88 & 0.92 \\
\hline
\end{tabular}

Figure 1 shows the ROC curves for ADC, CDI, and SCDI.

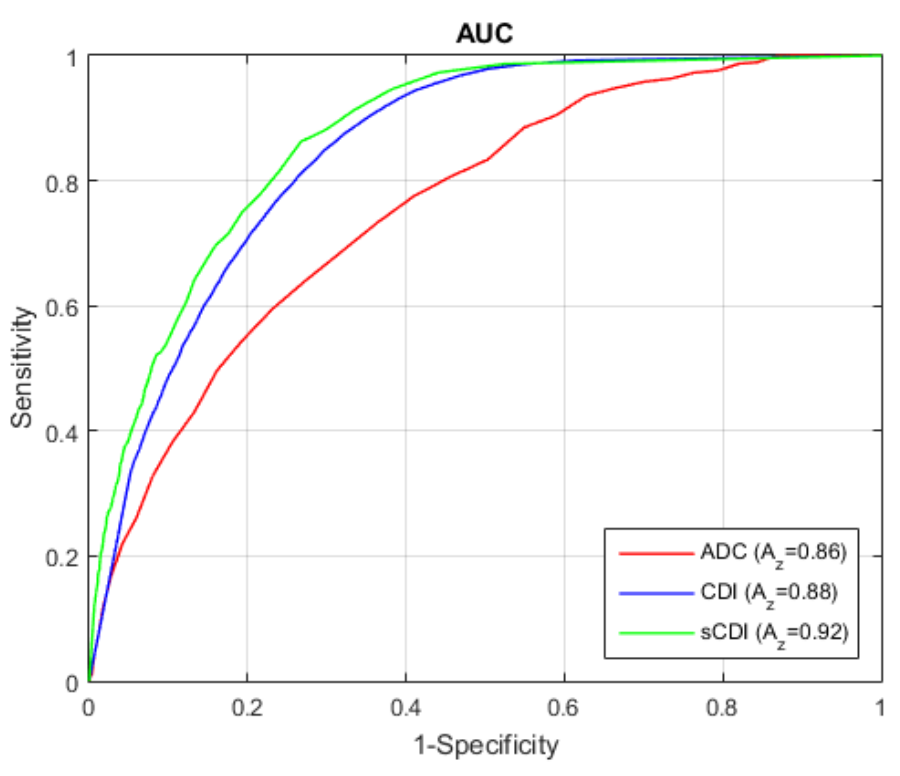

Fig. 1: ROC curves and the area under ROC curves $\left(A_{z}\right)$ for ADC, CDI, sCDI.

Figure 2 shows a confirmed prostate tumour in (a) ADC, (b) $\mathrm{CDI}$, and (c) sCDI. As it can be seen, the tumour region is more distinguishable in SCDI compared to $\mathrm{CDI}$ and $\mathrm{ADC}$, with respect to surrounding healthy regions in the image. A better separation of cancerous and healthy tissue can be beneficial to both qualitative and quantitative assessments of prostate cancer. It can help the clinicians with more accurate qualitative assessment of prostate cancer and potentially reduce the inter-observer variability. In addition, visually different regions, as seen in $\mathrm{SCDI}$, usually translate to quantitative imaging features which belong to different classes of features. Hence, improved separability of cancerous regions in prostate in sCDI can also aid computer-aided detection algorithms for prostate cancer where imaging features are used to classify the pixels into cancerous and non-cancerous regions.

\section{Conclusion}

In this paper, sparse correlated diffusion imaging (SCDI) was introduced as a computational diffusion MRI (CD-MRI) modality. SCDI improves the separability of cancerous and healthy tissue in prostate gland by selecting an optimally sparse subset of diffusion signals and mixing them together. It was shown that sCDI is able to improve the delineation of cancerous regions and healthy regions in prostate. The improved ability to separate cancerous and healthy regions in prostate using SCDI can improve diagnostic accuracy and reduce the need for invasive methods such as biopsy, which is both harmful and painful. In addition, the ability to optimize sCDI using only PI-RADS score and radiologist's contours eliminates 


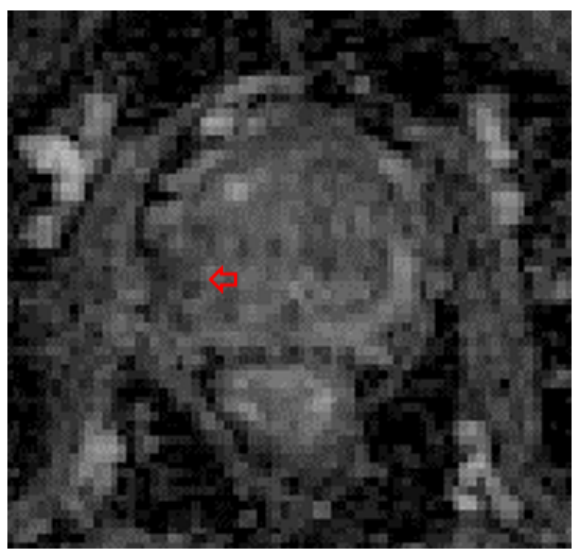

(a) ADC

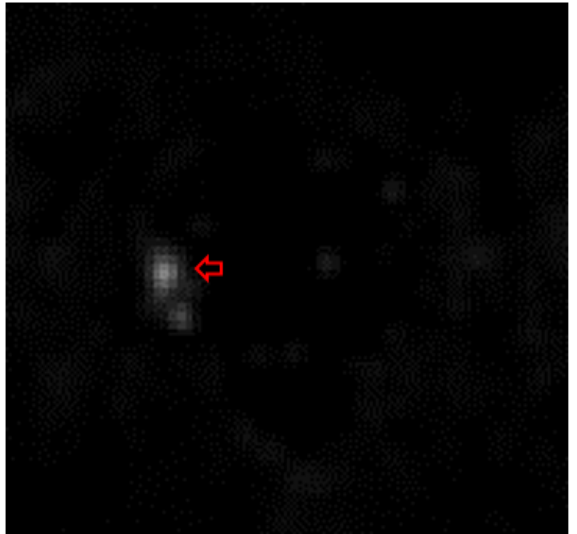

(b) $\mathrm{CDI}$

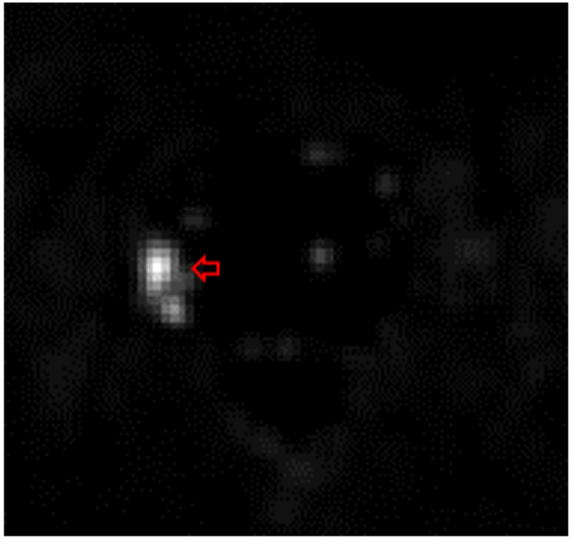

(c) eCDI

Fig. 2: A confirmed prostate tumour in (a) ADC, (b) CDI, (c) SCDI, shown by an arrow. The tumour region is more distinguishable in sCDI.

the need for wholemount or biopsy specimens as training data, which are difficult to obtain. As a result, SCDI has the potential to be configured and optimized easily and integrated into mpMRI or computer-aided detection algorithms for prostate cancer, helping to achieve high accuracy diagnostics for prostate cancer.

\section{Acknowledgments}

This work was supported by the Natural Sciences and Engineering Research Council (NSERC) of Canada, Ontario Ministry of Research and Innovation, Ontario Institute for Cancer Research, and in part by the Canada Research Chairs program.

\section{References}

[1] Canadian Cancer Society, "Canadian Cancer Statistics," Tech. Rep., 2015.

[2] C. A. Woodfield, G. A. Tung, D. J. Grand, J. A. Pezzullo, J. T. Machan, and J. F. Renzulli, "Diffusion-weighted MRI of peripheral zone prostate cancer: Comparison of tumor apparent diffusion coefficient with Gleason score and percentage of tumor on core biopsy," International Braz J Urol, vol. 36, no. 4, p. 504, 2010.

[3] J. O. Barentsz, J. Richenberg, R. Clements, P. Choyke, S. Verma, G. Villeirs, O. Rouviere, V. Logager, and J. J. Fütterer, "ESUR prostate MR guidelines 2012," European Radiology, vol. 22, no. 4, pp. 746-757, 2012.

[4] B. G. Muller, J. H. Shih, S. Sankineni, J. Marko, S. RaisBahrami, A. George, J. J. M. C. H. de la Rosette, M. J. Merino, B. J. Wood, P. Pinto, P. L. Choyke, and B. Turkbey, "Prostate Cancer: Interobserver Agreement and Accuracy with the Revised Prostate Imaging Reporting and Data System at Multiparametric MR Imaging." Radiology, no. May 2016, p. 142818, 2015. [Online]. Available: http://www.ncbi.nlm.nih.gov/pubmed/26098458

[5] A. Wong, J. Glaister, A. Cameron, and M. Haider, "Correlated diffusion imaging," BMC medical imaging, vol. 13, p. 26, 2013.

[6] F. Khalvati, A. Modhafar, A. Cameron, A. Wong, and A. Haider, "A Multi-Parametric Diffusion Magnetic Resonance Imaging
Texture Feature Model for Prostate Cancer Analysis," in International Conference on Medical Image Computing and Computer Assisted Intervention (MICCAI), Computational Diffusion MRI, 2014, pp. 79-88.

[7] F. Khalvati, A. Wong, and M. A. Haider, "Automated prostate cancer detection via comprehensive multi-parametric magnetic resonance imaging texture feature models." $B M C$ medical imaging, vol. 15, no. 1, p. 27, 2015. [Online]. Available: http://www.biomedcentral.com/1471-2342/15/27

[8] A. Cameron, F. Khalvati, M. Haider, and A. Wong, "MAPS: A Quantitative Radiomics Approach for Prostate Cancer Detection." IEEE transactions on bio-medical engineering, vol. 63, no. 6, pp. 1145-1156, 2016. [Online]. Available: http://www.ncbi.nlm.nih.gov/pubmed/26441442

[9] A. Wong, F. Khalvati, and M. A. Haider, "Dual-Stage Correlated Diffusion Imaging," in IEEE International Symposium on Biomedical Imaging, 2015, pp. 75-78.

[10] J. Zhang, A. Eilaghi, M. A. Haider, A. Wong, and F. Khalvati, "Optimized Correlated Diffusion Imaging for Prostate Cancer Detection," in Imaging Network Ontario (ImNO), 2016, p. 37.

[11] F. Khalvati, J. Zhang, M. A. Haider, and A. Wong, "Enhanced Dual-Stage Correlated Diffusion Imaging," in International Conference of the IEEE Engineering in Medicine and Biology Society (EMBC), 2016, pp. 5537-5540.

[12] J. Glaister, A. Cameron, A. Wong, and M. A. Haider, "Quantitative investigative analysis of tumour separability in the prostate gland using ultra-high b-value computed diffusion imaging," Proceedings of the Annual International Conference of the IEEE Engineering in Medicine and Biology Society, EMBS, pp. 420-423, 2012. 